\title{
Assistência de Enfermagem Perioperatória aos Pacientes com Câncer Colorretal: Caracterização Sociodemográfica, Clínica e Terapêutica
}

Perioperative Nursing Care for Patients with Colorectal Cancer: Sociodemographic, Clinical and Therapeutic Characterization

Atención de Enfermería Perioperatoria para Pacientes con Cáncer Colorrectal: Caracterización Sociodemográfica, Clínica y Terapéutica

André Aparecido da Silva Teles ORCID: https://orcid.org/0000-0002-0548-9592 Universidade de São Paulo, Brasil E-mail: andreteles@usp.br

Lorena Alves Pantoni ORCID: https://orcid.org/0000-0003-4542-3472 Universidade de São Paulo, Brasil E-mail: lorenapantoni@usp.br

Wagner Felipe dos Santos Neves ORCID: https://orcid.org/0000-0001-9989-7273 Universidade de São Paulo, Brasil E-mail: wagnerfelipeneves@usp.br Janderson Cleiton Aguiar ORCID: https://orcid.org/0000-0002-6095-8689 Universidade de São Paulo, Brasil E-mail: jcaguiar@usp.br

Tatiana Mara da Silva Russo ORCID: https://orcid.org/0000-0002-8077-0595 e-mail: tatiana.russo@usp.br

Camila Maria Silva Paraizo Horvath ORCID: https://orcid.org/0000-0002-3574-7361 E-mail: camilaparaizo@usp.br

Antonio Jorge Silva Correa Júnior ORCID: https://orcid.org/0000-0003-1665-1521

Universidade de São Paulo, Brasil.

E-mail: antoniocorreajunior@usp.br Helena Megumi Sonobe

ORCID: https://orcid.org/0000-0003-3722-0835

Universidade de São Paulo, Brasil E-mail: megumi@eerp.usp.br

\begin{abstract}
Resumo
Objetivo: caracterizar o perfil sociodemográfico, clínico e terapêutico dos pacientes, em sua primeira cirurgia eletiva por câncer colorretal, assim como da realização da demarcação pré-operatória do estoma, em um hospital universitário público paulista. Metodologia: estudo descritivo, retrospectivo e transversal, com abordagem quantitativa, por meio de levantamento de prontuários, com um instrumento de coleta de dados sobre o perfil sociodemográfico, clínico e terapêutico de pacientes da especialidade Coloproctologia, em sua primeira cirurgia eletiva por câncer colorretal. Resultados: houve predomínio de idosos, com companheiros, ensino fundamental, aposentados, localização do tumor na junção sigmóide, retossigmóide e retal, presença de comorbidades e sobrepeso/obesidade, adenocarcinoma moderadamente diferenciado, cirurgias realizadas por cirurgia aberta, hemilectomia/retossigmoidectomia A maioria não realizou tratamento neoadjuvante e todos os participantes receberam demarcação pré-operatória do estoma. Após a cirurgia, $51(42,1 \%)$ pacientes apresentaram predominantemente colostomia, todos contrarreferenciados ao programa especializado do Sistema Único de Saúde (SUS). Conclusão: Os resultados evidenciaram a importância da sistematização da demarcação do estoma, os aspectos fundamentais que influenciaram na demarcação do estoma, bem como na avaliação clínica, indicando a necessidade de capacitação especializada dos enfermeiros generalistas das instituições hospitalares sobre demarcação de estoma e assistência perioperatória fundamental para estes pacientes, com vistas à prevenção de complicações de estoma e da pele periestomal.

Palavras-chave: Perfil de saúde; Neoplasias colorretais; Estomas cirúrgicos; Enfermagem perioperatória; Estomaterapia.
\end{abstract}




\begin{abstract}
Objective: to characterize the sociodemographic, clinical and therapeutic profile of two patients, in their first elective surgery for coloretal cancer, as well as the realization of the pre-operative demarcation of the stoma, in a São Paulo public university hospital. Methodology: descriptive, retrospective and cross-sectional study, with a quantitative approach, by means of the collection of medical records, with a data collection instrument on the sociodemographic, clinical and therapeutic profile of patients of the Coloproctology specialty, in their first elective surgery for coloretal cancer. Results: there is a predominance of idosos, with companions, fundamental ensino, settled, location of the tumor in the sigmoid junction, sigmoid and retal challenges, presence of comorbidities and overweight / obesity, moderately differentiated adenocarcinoma, surgeries performed by open surgery, neural hemilemoid surgery / challenges Perform neoadjuvant treatment for all participants to receive stoma pre-operative demarcation. After surgery, $51(42.1 \%)$ patients presented predominantly colostomy, all cross-referenced to a specialized program of the Brazilian Public Health System (SUS). Conclusion: The results show the importance of the systematization of the stoma demarcation, the fundamental aspects that influence the demarcation of the stoma, as well as the clinical assessment, indicating the need for specialized training by two general nurses from hospital institutions on stoma demarcation and fundamental perioperative care for these patients, regarding the prevention of stoma complications and the peristomal skin.
\end{abstract}

Keywords: Health profile; Colorectal neoplasms; Surgical stomas; Perioperative nursing; Stomatherapy.

\title{
Resumen
}

Objetivo: caracterizar el perfil sociodemográfico, clínico y terapéutico de los pacientes en su primera cirugía electiva por cáncer colorrectal, así como la demarcación preoperatoria del estoma, en un hospital universitario público de São Paulo. Metodología: estudio descriptivo, retrospectivo y transversal, con abordaje cuantitativo, a través de la encuesta de historias clínicas, con un instrumento de recolección de datos sobre el perfil sociodemográfico, clínico y terapéutico de los pacientes de la especialidad de Coloproctología, en su primera cirugía electiva. para el cáncer colorrectal. Resultados: hubo predominio de ancianos, con pareja, educación básica, jubilados, localización tumoral en la unión sigmoidea, rectosigmoidea y rectal, presencia de comorbilidades y sobrepeso / obesidad, adenocarcinoma moderadamente diferenciado, cirugías realizadas por cirugía abierta, hemilectomía / rectosigmoidectomía La mayoría no se sometió a tratamiento neoadyuvante y todos los participantes recibieron la demarcación preoperatoria del estoma. Después de la cirugía, $51(42,1 \%)$ pacientes tuvieron predominantemente colostomía, todos los cuales no fueron referidos al programa especializado del Sistema Único de Salud (SUS). Conclusión: Los resultados mostraron la importancia de sistematizar la demarcación del estoma, los aspectos fundamentales que incidieron en la demarcación del estoma, así como la evaluación clínica, indicando la necesidad de formación especializada de enfermeras generalistas en instituciones hospitalarias en demarcación de estoma y asistencia perioperatoria fundamental para estos pacientes, con el fin de prevenir complicaciones de la piel estoma y periestomal.

Palabras clave: Perfil de salud; Neoplasias colorrectales; Estomas quirúrgicos; Enfermería perioperatória; Estomaterapia.

\section{Introdução}

Conceitualmente, o termo estomia ou estoma, de origem grega, é a abertura artificial, confeccionada cirurgicamente em um órgão oco, para comunicação com o meio externo. No caso da porção intestinal, a sua finalidade é o desvio do trânsito para eliminação dos resíduos fecais (Rocha, 2011; Santos \& Cesaretti, 2015). Vários diagnósticos como doenças diverticulares, doenças inflamatórias intestinais (Doença de Crohn e Retocolite ulcerativa) e, principalmente, as neoplasias que acometem o sistema digestório, podem resultar no tratamento cirúrgico com confecção de um estoma intestinal. Acrescem-se outras situações como traumas abdominais e anomalias colorretais, que podem também necessitar deste tratamento (Rocha, 2011; Habr-Grama; Scanavini Neto; Araújo, 2015).

O Câncer Colorretal (CCR) é a principal causa de confecção de estomias intestinais (Santos, 2006). Um estudo global, publicado recentemente, demonstrou que, somente no ano de 2017, foram diagnosticados mais de 1.833 .000 casos de CCR no mundo (Fitzmaurice et al., 2019). A cirurgia é a principal alternativa terapêutica para o CCR, o que envolve a possibilidade de confecção de estomia intestinal, assim como para as outras doenças colorretais mencionadas. A estomização constitui um desafio para os profissionais de saúde, devido ao número crescente de pacientes, que necessitarão desta confecção, quer seja em caráter temporário, que possibilita a reconstrução do trânsito intestinal ou definitiva, quando esta condição não é reversível (Rocha, 2011). 
A American Society of Colon and Rectal Surgeons (ASCRS) e a Wound Ostomy and Continence Nurses Society (WOCN) recomendam a realização da demarcação de estoma pré-operatória por estomaterapeuta ou enfermeiro com treinamento especializado, em todos os pacientes submetidos às cirurgias com possibilidade de confecção de estoma (Wound, Ostomy, and Continence Nurses Society, 2007; 2010). Portanto, a demarcação de estoma é uma intervenção estrita do enfermeiro estomaterapeuta ou enfermeiro capacitado parar assegurar a localização mais apropriada do estoma para o paciente, favorecendo o autocuidado, a redução de complicações precoces e tardias, que deverá estar vinculada ao ensino pré-operatório. Existem vários aspectos, que devem ser analisados durante esta demarcação, para possibilitar a aplicação e a utilização adequada dos equipamentos coletores e adjuvantes (Wound, Ostomy, and Continence Nurses Society, 2007; 2010; Oliveira, 2014; World Council of Enterostomal Therapists, 2014; Sonobe, 2017).

O processo de estomização traz repercussões na vida familiar, social, laboral, financeira e psicoemocional destes pacientes e seus familiares, o que requer assistência perioperatória multiprofissional e a Enfermagem tem exercido um papel importante, que é reconhecido por pacientes, familiares e por outros profissionais da saúde (Martins et al., 2015; Shun et al., 2016; Wiendenbein et al., 2016; Teles et al., 2017; Yu et al., 2017; Mcmullen et al., 2018; Sasaki, 2018). Assim, a assistência de enfermagem perioperatória pressupõe o ensino pré-operatório do paciente e familiar sobre a cirurgia e suas consequências, demarcação pré-operatória do local do estoma, preparo colônico e identificação de fatores de risco para prevenção de complicações pós-operatórias, com a inserção da família no planejamento de cuidado de Enfermagem. E no pós-operatório, para o paciente com estoma, a avaliação da evolução clínica e do estoma, bem como a retomada do ensino do autocuidado sobre os cuidados específicos com o estoma e os equipamentos coletores, principalmente para a família, que inicialmente será o principal cuidador domiciliário. No contexto brasileiro, deve-se assegurar a contrarreferência para cadastramento deste paciente no Programa de Ostomizados do Sistema Único de Saúde (SUS) em nível secundário, na perspectiva da Alta Responsável (Brasil, 2009; Brasil, 2013; Oliveira, 2014; Sonobe, 2017; Teles et al., 2017).

Ressalta-se que a demarcação de estoma é o ponto inicial para a reabilitação do paciente, que deverá ter a acessibilidade às políticas públicas de saúde, que dependem da implementação da Portaria $\mathrm{N}^{\circ} 400$, em 16 de novembro de 2009, que estabeleceu a legislação atual brasileira sobre a organização e dinâmica de atendimento dos estomizados, com definição dos fluxos de referência e contrarreferência, integrando os diferentes níveis de atenção, com as Diretrizes Nacionais para a Atenção à Saúde das Pessoas Estomizadas no SUS, em todas as unidades federadas, respeitadas as competências das três esferas de gestão (Brasil, 2009).

Diante do exposto, considera-se importante o conhecimento sobre as características sociodemográficas, clínicas e terapêuticas destes pacientes para subsidiar o planejamento da assistência de enfermagem e a importância da demarcação de estoma pelo enfermeiro estomaterapeuta ou enfermeiro capacitado, as questões de pesquisa formuladas foram: Qual é a caracterização sociodemográfica, clínica e terapêutica de pacientes em sua primeira cirurgia eletiva por câncer colorretal? Estes pacientes têm recebido a demarcação de estoma pré-operatória?

Para responder estas questões, estabeleceu-se como objetivo deste estudo caracterizar o perfil sociodemográfico, clínico e terapêutico dos pacientes, em sua primeira cirurgia eletiva por câncer colorretal, assim como da realização da demarcação pré-operatória do estoma, em um hospital universitário público paulista.

\section{Metodologia}

\subsection{Aspectos éticos}

O projeto de pesquisa, elaborado de acordo com os preceitos éticos da Resolução do Conselho Nacional de Saúde ${ }^{\circ}$ 466 de 12 dezembro de 2012, foi aprovado pelo Comitê de Ética em Pesquisa (CEP) da Instituição proponente, via Plataforma Brasil, sob número CAAE: 83343818.7.0000.5393. 
Para minimização dos riscos, os dados foram coletados mediante ao acesso aos prontuários eletrônicos rotineiramente preenchidos na instituição por todos os membros da equipe multiprofissional, assim como identificou-se os prontuários por códigos, assegurando o anonimato dos participantes do estudo e o sigilo dos dados confidenciais e privativos.

\subsection{Descrição do estudo}

Trata-se de um estudo descritivo, retrospectivo e de corte transversal, de abordagem quantitativa, por meio de levantamento de prontuários, sobre o perfil sociodemográfico, clínico e terapêutico dos pacientes da especialidade da Coloproctologia de uma Instituição de Ensino Superior Pública paulista, em sua primeira cirurgia eletiva por CCR. No estudo transversal, todas as medições são feitas em uma única ocasião (Hulley; Cummings; Browner; Grady; Newman, 2015).

\subsection{Local do estudo}

O local do estudo foi um Hospital Universitário, de nível terciário e quaternário, instituído em 1956, pela Lei Estadual 3.724 de 23/12/1955. Este constitui referência regional para o atendimento dos 26 municípios da Divisão Regional de Saúde (DRS) pelo SUS, além de atender pelo sistema de referência, outras cidades do Estado de São Paulo e de outras regiões do Brasil (Hospital Das Clínicas Da Faculdade De Medicina De Ribeirão Preto Da Universidade De São Paulo, 2020).

No ano de 2018, foram realizados 720.664 atendimentos, 35.418 internações e 28.425 cirurgias, além de 1.594 partos e 283 transplantes por esta Instituição, que atualmente integra um Complexo Acadêmico de Saúde vinculada à uma Instituição de Ensino Superior Pública (Hospital Das Clínicas Da Faculdade De Medicina De Ribeirão Preto Da Universidade De São Paulo, 2020).

A especialidade da Coloproctologia conta, atualmente, com 11 leitos, alocados nas Unidades de Internação Cirúrgica e de Oncologia Cirúrgica.

\subsection{População e amostra do estudo}

Para a definição da população do estudo, realizou-se o levantamento de todas as cirurgias eletivas realizadas pela especialidade da Coloproctologia no ano de 2019. Os critérios de inclusão foram: prontuários de pacientes com mais de 18 anos, atendidos pela Coloproctologia da Instituição, onde o estudo foi desenvolvido, nos meses de janeiro a dezembro de 2019, que passaram pela primeira vez pelo tratamento cirúrgico eletivo por CCR.

Foram excluídos os prontuários de pacientes que passaram por cirurgia de urgência, que já eram estomizados ou que apresentavam recidiva por CCR. Assim, no ano de 2019, foram realizadas 308 cirurgias eletivas na especialidade da Coloproctologia e, destes, 121 pacientes (100\%) atenderam aos critérios de inclusão, constituindo a amostra deste estudo.

\subsection{Procedimentos para coleta de dados}

Os dados foram coletados nos meses de janeiro e fevereiro de 2020, após a definição dos prontuários elegíveis, cujos dados sobre a evolução clínica e da ficha operatória foram coletados nos sistemas informatizados e do Prontuário Eletrônico do Paciente.

Um instrumento de coleta de dados foi elaborado pelos pesquisadores, que contemplou idade, sexo, raça, procedência, escolaridade, situação profissional, estado civil, peso, altura, Índice de Massa Corporal (IMC), presença de comorbidades e cirurgias abdominais prévias, tempo de internação, diagnóstico médico, data do diagnóstico, tratamentos realizados, início do tratamento, cirurgia realizada, tipo de cirurgia, tipo histológico do tumor, demarcação pré-operatória do local do estoma e tipo de estoma intestinal confeccionada. 


\subsection{Variáveis do estudo}

As variáveis definidas para este estudo foram: Idade; Sexo; Raça; Procedência; Peso; Altura; IMC; Escolaridade; Situação laboral; Estado civil; Presença de comorbidades e cirurgias abdominais prévias; Data do diagnóstico; Localização primária: corresponde à localização anatômica do tumor; Data do início do tratamento; Tratamento Neoadjuvante realizado; Tempo de internação; Cirurgia realizada; Tipo de cirurgia: Aberta (Laparotomia), Videolaparoscópica; Tipo histológico do tumor; Demarcação pré-operatória do local do estoma; Tipo e local de estoma intestinal confeccionado.

\subsection{Análise dos dados}

Elaborou-se um de banco de dados Microsoft Excel®, mediante dupla digitação com verificação da consistência dos dados e sua consolidação. A análise descritiva foi realizada mediante as médias, medianas e desvio-padrão para as variáveis quantitativas; e frequências absoluta e percentual para variáveis categóricas, com utilização do programa IBM-SPSS® for Windows versão 22.0.

\section{Resultados}

A caracterização sociodemográfica da amostra deste estudo, constituída por 121 participantes, encontra-se na Tabela 1.

Pode-se verificar que a maioria dos pacientes era proveniente do Estado de São Paulo (95,9\%), sendo que 37,2\% eram da cidade do estudo.

Tabela 1. Caracterização sociodemográfica dos 121 pacientes segundo sexo, idade, raça, estado civil, escolaridade e situação laboral. Ribeirão Preto, SP, Brasil, 2021.

\begin{tabular}{|l|l|l|l}
\hline \multicolumn{2}{l}{ Variável } & \multicolumn{1}{c}{ n (\%) } & \multicolumn{1}{c}{ Média (DP)* } \\
\hline Sexo & Feminino & $63(52,1)$ & \\
\hline Idade & Masculino & $58(47,9)$ & $63(12,5)$ \\
\hline Raça & & & \\
\hline & Branca/Amarela & $102(84,3)$ & \\
\hline Estado Civil & Preta/Parda & $19(15,7)$ & \\
\hline Escolaridade & Com Companheiro & $63(52,1)$ & \\
\hline & Sem Companheiro & $58(47,9)$ & \\
\hline & Nenhuma & $5(4,2)$ & \\
\hline Situação laboral & Ensino Fundamental & $73(60,3)$ & \\
\hline & Ensino Médio & $30(24,8)$ & \\
\hline & Ensino Superior & $13(10,7)$ & \\
\hline & Ativo & $28(23,1)$ & \\
\hline & Aposentado & $62(51,2)$ & \\
\hline & Afastado & $10(8,3)$ & \\
\hline & Inativo/desempregado & $6(5,0)$ & \\
\hline & Do lar & $15(12,4)$ & \\
\hline & & &
\end{tabular}

Média (DP)* = Média (Desvio-Padrão). Fonte: Dados dos autores.

$\mathrm{Na}$ Tabela 2, tem-se a caracterização clínica dos pacientes do estudo, no qual observa-se que a localização do tumor na região do reto, junção retossigmoide, sigmoide e canal anal ocorreu em $84(69,4 \%)$ pacientes, assim como as variáveis sobrepeso e obesidade foram constadas em 63 (52\%) pacientes. Ainda, houve a presença de comorbidades físicas com 182 indicações, ou seja, mais que uma comorbidade por cada participante. Dentre as outras comorbidades, destacaram-se 
Ansiedade, Depressão, Insuficiência Renal Crônica (IRC) não dialítica e outras neoplasias. Na amostra, 63 (52,1\%) pacientes haviam realizado cirurgia abdominal prévia.

Tabela 2. Caracterização clínica dos 121 pacientes segundo localização do CCR, presença de comorbidades e IMC. Ribeirão Preto, SP, Brasil, 2021.

\begin{tabular}{|c|c|c|c|}
\hline \multicolumn{2}{|r|}{ Variável } & $\mathbf{n}$ & $\%$ \\
\hline \multicolumn{4}{|c|}{ Localização do CCR } \\
\hline & Reto & 32 & 26,4 \\
\hline & Junção Retossigmoide & 27 & 22,3 \\
\hline & Sigmoide & 23 & 19,0 \\
\hline & Cólon Ascendente & 14 & 11,6 \\
\hline & Ceco & 11 & 9,1 \\
\hline & Cólon Descendente & 9 & 7,4 \\
\hline & Cólon Transverso & 2 & 1,7 \\
\hline & Canal anal & 2 & 1,7 \\
\hline & Reto alto e Cólon Transverso (Sincrônicos) & 1 & 0,8 \\
\hline \multicolumn{4}{|c|}{ Presença de Comorbidades } \\
\hline & Hipertensão Arterial Sistêmica & 67 & 55,4 \\
\hline & Diabetes Mellitus & 26 & 21,5 \\
\hline & Dislipidemia & 14 & 11,6 \\
\hline & Anemia & 12 & 9,9 \\
\hline & Outras Comorbidades & 63 & 52,1 \\
\hline \multirow[t]{5}{*}{ IMC } & Baixo Peso & 9 & 7,4 \\
\hline & Eutrofia & 40 & 33,1 \\
\hline & Sobrepeso & 32 & 26,5 \\
\hline & Obesidade & 31 & 25,6 \\
\hline & Pacientes com dados de IMC Omissos no Sistema & 9 & 7,4 \\
\hline
\end{tabular}

Fonte: Dados dos autores.

$\mathrm{Na}$ Tabela 3 estão representados os tipos histológicos das lesões, identificados mediante à análise anatomopatológica do material coletado para biópsia no intraoperatório, identificando-se o predomínio de pacientes com Adenocarcinoma moderadamente diferenciado, o que indicou uma média capacidade invasiva deste tumor em relação aos tecidos adjacentes.

Tabela 3. Caracterização dos 121 pacientes segundo o tipo histológico. Ribeirão Preto, SP, Brasil, 2021.

\begin{tabular}{|c|c|c|}
\hline \multicolumn{1}{|c|}{ Tipo Histológico } & N & $\mathbf{\%}$ \\
\hline Adenocarcinoma moderadamente diferenciado & 102 & 84,3 \\
\hline Outros tipos & 19 & 15,7 \\
\hline Total & 121 & 100,0 \\
\hline
\end{tabular}

Fonte: Dados dos autores.

$\mathrm{Na}$ Tabela 4 está demonstrada a caracterização terapêutica dos participantes do estudo, sendo que as técnicas cirurgias convencionais (via aberta) predominaram condizentes com comprometimento clínico destes participantes, apesar de a maioria não ter realizado tratamento neoadjuvante, o que pode ser corroborado com os dados de tempo de internação que, em média, foi de 9,74 (Mediana=7, DP=7,6) dias, sendo o mínimo de dois e o máximo de 42 dias.

Acresce-se que ainda, que foi verificado para os pacientes que realizaram o tratamento neoadjuvante, o tempo decorrido entre o diagnóstico e o início do tratamento, cuja média foi de 75 dias (Mediana=66, DP=43,3). Quando a terapêutica de primeira linha foi a cirurgia, este tempo foi de 30,5 (Mediana=19, DP=36,3) dias. 
Tabela 4. Caracterização terapêutica dos 121 pacientes segundo tratamento neoadjuvante, demarcação pré-operatória e procedimento cirúrgico realizado. Ribeirão Preto, SP, Brasil, 2021.

\begin{tabular}{|c|c|c|}
\hline Terapêuticas & $\mathbf{n}$ & $\%$ \\
\hline \multicolumn{3}{|l|}{ Tratamento Neoadjuvante } \\
\hline \begin{tabular}{|l|l} 
Não &
\end{tabular} & 111 & 91,7 \\
\hline Sim & 10 & 8,3 \\
\hline \multicolumn{3}{|l|}{ Tipo de Tratamento } \\
\hline \begin{tabular}{|l|l} 
& Quimioterapia e Radioterapia
\end{tabular} & 7 & 70,0 \\
\hline Radioterapia & 2 & 20,0 \\
\hline Quimioterapia & 1 & 10,0 \\
\hline Demarcação pré-operatória & 121 & 100,0 \\
\hline \multicolumn{3}{|l|}{ Cirurgia Realizada } \\
\hline \begin{tabular}{|l|l} 
& Hemicolectomia
\end{tabular} & 49 & 40,5 \\
\hline Retossigmoidectomia & 35 & 28,9 \\
\hline Colectomia total & 9 & 7,4 \\
\hline Sigmoidectomia & 7 & 5,8 \\
\hline Amputação Abdominoperineal de reto & 6 & 5,0 \\
\hline Ressecção transanal de lesão de reto & 6 & 5,0 \\
\hline Colostomia Paliativa & 4 & 3,3 \\
\hline Laparotomia Exploradora & 4 & 3,3 \\
\hline Exanteração pélvica & 1 & 0,8 \\
\hline \multicolumn{3}{|l|}{ Tipo de Cirurgia } \\
\hline Aberta & 70 & 57,9 \\
\hline Videolaparoscópica & 45 & 37,2 \\
\hline Orificial & 6 & 5,0 \\
\hline Total & 121 & 100,0 \\
\hline
\end{tabular}

Fonte: Dados dos autores.

Pode-se verificar na Tabela 5, que todos os pacientes do estudo receberam a demarcação pré-operatória do estoma, sendo que para 51(42,1\%) pacientes, o tratamento cirúrgico resultou na confecção de estomia intestinal.

Dentre os tipos de estoma, predominou a colostomia, mas ressalta-se dois casos nos quais foram confeccionados colostomia úmida e um caso com uma colostomia e uma urostomia distintamente, além de três casos com colostomia perineal. Ainda, deve-se atentar para os estomas confeccionados do lado D, pois estes envolvem ileostomias e de transversostomias. Na alta hospitalar, todos os pacientes com estoma foram contrarreferenciados ao Programa de Ostomizados, mantido pelo SUS.

Tabela 5. Caracterização dos 51 pacientes segundo o tipo e a localização do estoma intestinal confeccionado. Ribeirão Preto, SP, Brasil, 2021.

\begin{tabular}{|c|c|c|}
\hline Variável & $\mathbf{N}$ & $\%$ \\
\hline \multicolumn{3}{|l|}{ Tipo de Estoma } \\
\hline \begin{tabular}{|l|l} 
Colostomia \\
\end{tabular} & 39 & 76,6 \\
\hline Ileostomia & 9 & 17,7 \\
\hline Colostomia Úmida & 2 & 3,8 \\
\hline Colostomia + Urostomia & 1 & 1,9 \\
\hline \multicolumn{3}{|l|}{ Localização do Estoma } \\
\hline \begin{tabular}{|l|l|} 
Lado E \\
\end{tabular} & 27 & 52,9 \\
\hline Lado D & 21 & 41,2 \\
\hline Perineal & 3 & 5,9 \\
\hline Total & 51 & 100,0 \\
\hline
\end{tabular}

E- Esquerdo D- Direito. Fonte: Dados dos autores. 


\section{Discussão}

Segundo a estimativa do Instituto Nacional de Câncer José Alencar Gomes da Silva (2019), o CCR é mais incidente nos homens, porém, neste estudo, houve predomínio de mulheres, corroborando os resultados anteriores de Oliveira (2014), realizado no local deste estudo, no qual foi analisado o seguimento realizado por pacientes por três anos, submetidos à cirurgia em 2009, por meio do levantamento de prontuários, focalizando-se as complicações de estoma e de pele periestoma. Por outro lado, o estudo de Carvalho (2014) sobre a sobrevida de pacientes diagnosticados com CCR no período de 2000 a 2010, com levantamento de dados pelo Registro Hospitalar do Câncer nesta Instituição, identificou que 51,3\% eram homens, enfatizandose que, somente foram cadastrados neste sistema, pacientes que efetivamente iniciaram o primeiro tratamento na Instituição. Pode-se inferir que o predomínio discreto de homens pode ser decorrente da busca tardia destes pela assistência à saúde.

$\mathrm{O}$ fato de a maioria possuir companheiro torna-se importante, pois a capacidade de enfrentamento do adoecimento e dos tratamentos de pacientes é fortalecida com a rede de apoio familiar e social, assim como pelo suporte multiprofissional, oferecido no contexto de atendimento à saúde (Gonzalez-Saenz de Tejada et al., 2017; Teles et al., 2017; Silva et al., 2017).

Há que se ressaltar, que a maioria dos participantes possuía até o ensino fundamental completo, o que pode influenciar no entendimento sobre as informações oferecidas pelos profissionais da saúde no perioperatório, principalmente no préoperatório quando é realizado o ensino para paciente e familiar sobre cirurgia e suas consequências, vinculados à demarcação de estoma; e no pós-operatório quando são retomados aspectos do ensino com canalização para o autocuidado com o estoma e equipamentos coletores e adjuvantes, além da contrarreferência ao Programa de Ostomizados (Pereira, et al., 2016). Este planejamento deve ser realizado pelo Enfermeiro, de forma integrada à equipe multiprofissional, sendo que este tem assumido o papel de coordenação das atividades profissionais no perioperatório (Shun et al., 2016).

Houve predomínio de idosos neste estudo, assim como em outros estudos (Carvalho, 2014; Oliveira, 2014), o que indica a necessidade de os profissionais incluírem a família para assegurar os cuidados domiciliários no pós-operatório para prevenção de complicações de ferida; prevenção de complicações de estoma e de pele periestoma, assim como o autocuidado com os equipamentos coletores e adjuvantes para os estomizados, além do seguimento de controle oncológico (Shahrokni et al., 2016; Bulkley et al., 2018; Sasaki, 2018).

O adenocarcinoma é a forma histológica mais comum do CCR, correspondendo a mais de $85 \%$ dos casos, sendo que este tipo histológico com a doença em fase avançada tem constituído um dos grandes desafios para os profissionais da saúde, considerando-se a complexidade para o alcance da resolução curativa para estes pacientes (Rocha, 2011; Oliveira, 2014; Carvalho, 2014). Isto requer maior eficiência na implementação das políticas públicas de saúde com integração da Atenção Primária a Saúde aos outros níveis de atendimento da população, bem como a implementação de diretrizes para a definição dos tratamentos e do seguimento de controle a ser realizado pela equipe multiprofissional, que deverá ter capacitação especializada, além de infraestrutura com maior densidade tecnológica (Brasil, 2014; Carvalho, 2014; Teles et al., 2017).

Acresce-se, ainda, uma discussão sobre a prevenção e promoção da saúde da população, por meio de programas para alimentação saudável, controle da obesidade e atividade física regular, além do rastreamento de pessoas com fatores de risco específicos como história pregressa de neoplasia colorretal e na família, doenças inflamatórias intestinais, doenças hereditárias como polipose familiar (Shun et al., 2016; Brasil, 2014; Oliveira, 2014, Carvalho, 2014).

No que se refere ao tratamento cirúrgico para $\mathrm{CCR}$, a demarcação de estoma pré-operatória tem sido reconhecida como um aspecto fundamental para a prevenção de complicações de estoma e de pele periestoma e assim, o IMC é um dado clínico importante para esta intervenção do enfermeiro estomaterapeuta ou do enfermeiro com esta capacitação (Wound Ostomy and Continence, 2010, Oliveira, 2014; Sonobe, 2017). Além disto, outras características clínicas como presença de cirurgia abdominal prévia e de hérnia incisional, tratamentos neoadjuvantes como quimioterapia antineoplásica e radioterapia, além da instabilidade hemodinâmica, metabólica e neurológica tem influenciado no estabelecimento do tratamento eletivo, 
bem como na evolução expectante para o paciente (Brasil, 2014; Oliveira, 2014).

A irradiação neoadjuvante na região perineal dos pacientes pode resultar em lesões de pele e dor, além de interferir no processo de cicatrização quando a cirurgia é realizada via perineal e na confecção da colostomia perineal. O enfermeiro estomaterapeuta ou enfermeiro com esta capacitação tem a responsabilidade pelo planejamento da assistência de enfermagem perioperatória, para assegurar o sucesso terapêutico para estes pacientes e seus familiares (Shun et al., 2016; Yu et al., 2017; Den Bakker et al., 2018; Mcmullen et al., 2018).

Ainda, para aqueles que realizaram o tratamento neoadjuvante, o tempo decorrido entre o diagnóstico e o início do deste tratamento foi, em média, de 75 dias, o que denota a dificuldade de implementação da Lei $\mathrm{N}^{\circ} 12.732 / 12$ em 22 de Novembro de 2012, sancionada pelo Governo Federal para o SUS, determinando o prazo máximo de 60 dias desde o estabelecimento do diagnóstico e prescrição da terapêutica pelo médico, até a realização dos tratamentos cirúrgicos, quimioterapia antineoplásica e/ou radioterapia. Contudo, quando não há necessidade do tratamento neoadjuvante, o tempo decorrido até a cirurgia é praticamente a metade do recomendado pela legislação, indicando um importante avanço nesta Especialidade Cirúrgica na Instituição do estudo (Brasil, 2012; Carvalho, 2014).

Com a análise do conjunto de dados, comprometimento clínico, localização do tumor oncológico nas regiões do reto, junção retossigmoide, sigmoide e canal anal e os procedimentos cirúrgicos Retossigmoidectomia, Sigmoidectomia, Amputação abdominoperineal de reto com confecção de estomas, Laparotomia explorada e Exanteração pélvica, realizados pelos participantes deste estudo, torna evidente a necessidade da acessibilidade à profissionais altamente especializados e também aos recursos de maior densidade tecnológica por estes pacientes. Para isso, há necessidade da implementação das políticas públicas de saúde com maior envolvimento dos profissionais, com integração dos diferentes contextos de atendimento na Rede de Atenção à Saúde do SUS (Wound Ostomy and Continence, 2010, Brasil, 2014; Carvalho, 2014; Oliveira, 2014; Sasaki et al., 2017; Sonobe, 2017; Teles et al., 2017)

A acessibilidade aos tratamentos neoadjuvantes e adjuvantes pelo SUS tem constituído um desafio, o que pode repercutir na evolução clínica, que por vezes, poderá inviabilizar o controle do CCR ou, mesmo, o alcance da cura pelo paciente, apesar do estabelecimento de diretrizes clínicas e terapêuticas para este diagnóstico para o SUS (Brasil, 2014; Carvalho, 2014; Shun et al., 2016).

Na realização da demarcação de estoma pré-operatória, há que se avaliar as repercussões dos tratamentos neoadjuvantes; as características da pele dos pacientes, abdômen com grande camada de tecido adiposo ou presença de pregas cutâneas; e capacidade do paciente para manter-se de pé, permanecer sentado ou em decúbito dorsal no leito para avaliação do local a ser demarcado, que influenciam a sua realização, além dos aspectos relativos à técnica de demarcação de estoma (Wound Ostomy and Continence, 2010, Oliveira, 2014; Sonobe, 2017).

Neste estudo, todos os pacientes com CCR, submetidos à primeira cirurgia eletiva por CCR, receberam a demarcação de estoma, fato que difere da realidade brasileira vigente. Do total de 121 participantes demarcados neste estudo, a confecção de estoma ocorreu em $42 \%$ destes, por considerar que a sistematização da demarcação pré-operatória para todos os pacientes, poderá minimizar a ocorrência de complicações de estoma e de pele periestoma. Desta forma, os enfermeiros estomaterapeutas têm sido valorizados na composição das equipes multiprofissionais, principalmente em relação à intervenção educativa para a prevenção e deteç̧ão precoce de CCR e seguimento especializado de pacientes com estomas por doença oncológica (Yu et al., 2017; Den Bakker et al., 2018; Mcmullen et al., 2018).

Neste estudo, os tipos de estoma da amostra relacionaram-se com a localização tumoral, que foram as regiões do reto, junção retossigmoide, sigmoide e canal anal, assim como ao tipo histológico adenocarcinoma moderadamente diferenciado, influenciando na manutenção desta condição por mais tempo ou mesmo em definitivo, principalmente quando analisados sob o ponto de vista da evolução e resposta clínica aos tratamentos antineoplásicos. 
Todos os pacientes com estoma foram contrarreferenciados ao Programa de Ostomizados do SUS, indicando a implementação da Portaria 400, e desta forma, asseguram-se a acessibilidade aos equipamentos coletores e adjuvantes e o seguimento multiprofissional para esta clientela, assim como a implementação da Alta Responsável (Brasil, 2009; Brasil, 2013). Considerando-se a complexidade clínica desta clientela, a assistência de enfermagem perioperatória necessita de maior investimento na educação permanente dos enfermeiros generalistas desta Instituição, no que se refere à avaliação pósoperatória do estoma e da pele periestoma, assim como no treinamento da demarcação de estoma. Esta intervenção tem sido realizada pelos enfermeiros residentes do Programa de Residência Multiprofissional em Atenção ao Câncer, que anualmente são capacitados pelos preceptores estomaterapeutas, vinculados à Instituição de ensino parceira.

\section{Conclusão}

Os resultados deste estudo sobre a caracterização sociodemográfica, clínica e terapêutica de pacientes com CCR em sua primeira cirurgia eletiva, indicaram a realização da demarcação de estoma em todos os participantes, predominantemente idosa, aposentada, com companheiros e ensino fundamental, localização tumoral na região sigmoide, junção retossigmoide e reto, presença de comorbidades e de sobrepeso/obesidade, adenocarcinoma moderadamente diferenciado, cirurgias Hemicolectomia/Retossigmoidectomia realizadas, por via aberta e ausência de tratamento neoadjuvante. Além disto, da amostra, 51(42,1\%) tiveram a confecção de estoma, com predomínio de colostomia e do posicionamento no lado E, sendo que todos foram contrarreferenciados ao programa especializado do SUS.

Estes resultados possibilitarão aperfeiçoar a assistência de enfermagem perioperatória ofertada, assim como a intervenção demarcação de estoma para esta clientela. A limitação do estudo foi a coleta de dados em um único momento por se tratar de um estudo transversal e pela impossibilidade de aprofundamento das variáveis clínicas por falta de registro de informações no prontuário eletrônico dos pacientes, principalmente no que se refere à exatidão da localização do estoma confeccionado e suas características, dados importantes para verificar a presença de complicações de estoma e de pele periestoma. Estudos futuros poderão explorar estas lacunas de registros clínicos e da avaliação de estoma e de pele periestoma, por meio de intervenções de educação permanente de enfermeiros nesta Instituição hospitalar.

\section{Referências}

Bulkley, J. E. et al. (2018). Ongoing ostomy self-care challenges of long-term rectal cancer survivors. Support Care Cancer. 26(11), 3933-3939. https://doi.org/10.1007/s00520-018-4268-0

Brasil. Instituto Nacional de Câncer José Alencar Gomes da Silva (INCA). (2019). Estimativa 2020: incidência de câncer no Brasil. https://www.inca.gov.br/publicacoes/livros/estimativa-2020-incidencia-de-cancer-no-brasil

Brasil. Ministério da Saúde. Secretaria de Atenção à Saúde. Portaria $\mathrm{n}^{\circ} \quad 400$, de $16 \quad$ de novembro de 2009 . http://bvsms.saude.gov.br/bvs/saudelegis/sas/2009/prt0400_16_11_2009.html

Brasil. Ministério da Saúde. (2012). Lei no 12.732, de 23 de Novembro de 2012.. http://www.planalto.gov.br/ccivil_03/_ato2011-2014/2012/lei/112732.htm.

Brasil. Ministério da Saúde. (2013). Portaria $\mathrm{n}^{\circ} \quad 3 . \quad 390$, de $30 \quad$ de 2013. http://bvsms.saude.gov.br/bvs/saudelegis/gm/2013/prt3390_30_12_2013.html

Brasil. Ministério da Saúde. (2014). Portaria $\mathrm{n}^{\mathrm{o}}$ 958, de $26 \quad$ de 2014 https://portalarquivos2.saude.gov.br/images/pdf/2014/novembro/24/MINUTA-Portaria-SAS-DDT-Colon-e-Reto-23-09-2014-corrigido-em-24-11-2014.pdf.

Carvalho, R. A. O. (2014). Análise do perfil epidemiológico e sobrevida de pacientes com câncer colorretal em um hospital universitário de 2000 a 2010. Dissertação - Escola de Enfermagem de Ribeirão Preto da Universidade de São Paulo. Ribeirão Preto-SP.

Den Bakker, C. M. et al. (2018). Cancer survivors' needs during various treatment phases after multimodal treatment for colon cancer - is there a role for eHealth? BMC Cancer. 18(1),1207. https://doi.org/10.1186/s12885-018-5105-z

Fitzmaurice, M. D. C. et al. (2019). Global, regional, and national cancer incidence, mortality, years of life lost, years lived with disability, and disabilityadjusted life-years for 29 cancer groups, 1990 to 2017: a systematic analysis for the global burden of disease study. Jama Oncology. 5(12), 749-1768. https://www.ncbi.nlm.nih.gov/pubmed/31560378 
Gonzalez-Saenz de Tejada, M. et al. (2017). Association between social support, functional status, and change in health-related quality of life and changes in anxiety and depression in colorectal cancer patients. Psychooncology. 26(9), 1263-1269. https://doi.org/10.1002/pon.4303

Habr-Grama, A., Scanavini Neto, A., Araújo, S. E. A. Estomias intestinais: aspectos conceituais e técnicos. In: Santos, V. L. C. G, Cesaretti, I. U. R. (2015). Assistência em estomaterapia: cuidando de pessoas com estomia. (2a ed.), Atheneu.

Hospital das Clínicas da Faculdade de Medicina de Ribeirão Preto da Universidade de São Paulo. HCFMRP-USP em números. https://site.hcrp.usp.br/hcfmrpem-numeros/

Hulley, S. B., Cummings, S. R., Browner, W. S.; Grady, D. G. \& Newman, T. B. (2015). Delineando a pesquisa clínica. (4a ed.) Artmed.

Martins, L. M. et al. (2015). Rehabilitation of individuals with intestinal ostomy. British Journal of Nursing. 24(22), S4-S11. https://doi.org/10.12968/bjon.2015.24.Sup22.S4

Mcmullen, C. et al. (2018). Designing for impact: identifying stakeholder-driven interventions to support recovery after major cancer surgery. Support Care Cancer. 26(12),4067-4076. https://www.ncbi.nlm.nih.gov/pubmed/29876832.

Oliveira, M. S. As complicações precoces e tardias e a demarcação de estoma intestinal. (2014). Dissertação (Mestrado) - Escola de Enfermagem de Ribeirão Preto da Universidade de

Pereira, A. C. et al. (2016). O ensino pré-operatório na perspectiva de pacientes oncológicos. Revista de Enfermagem UFPE online. 10, 449-456. https://doi.org/10.5205/1981-8963-v10i2a10976p449-456-2016

Rocha, J. J. R. Estomas intestinais (ileostomias e colostomias) e anastomoses intestinais. (2011). Medicina (Ribeirão Preto), 44(1),51-6. https://doi.org/10.11606/issn.2176-7262.v44i1p51-56

Sasaki, V. D. M. et al. Rehabilitation of people with intestinal stomy: integration review. Revista de Enfermagem UFPE online. 11(4), 1745-1754. https://doi.org/10.5205/1981-8963-v11i4a15271p1745-1754-2017

Sasaki, V. D. M. (2018). Autocuidado com a estomia intestinal e equipamentos coletores: perspectiva das pessoas estomizadas intestinais, familiares e equipe multidisciplinar do Programa de Ostomizados. Tese (Doutorado) - Escola de Enfermagem de Ribeirão Preto, Universidade de São Paulo. Ribeirão Preto-SP.

Santos, V. L. C. G. Cuidando do estomizados: análise da trajetória no ensino, pesquisa e extensão. (2006). Tese (Livre-Docência) - Escola de Enfermagem, Universidade de São Paulo,

Santos, V. L. C. G.; \& Cesaretti, I. U. R. Assistência em estomaterapia: cuidando de pessoas com estomia. (2015). (2a ed.) Atheneu.

Shahrokni, A. et al. (2017). How we care for an older patient with cancer. Journal Oncology Practice. 13(2), 95-102. 10.1200/JOP.2016.017608

Shun, S. C. et al. (2016). Cancer prehabilitation for patients starting from active treatment to surveillance. Asia Pac J Oncol Nurs. 3(1), 37-40. https://doi.org/10.4103/2347-5625.178169

Sonobe, H. M. (2017). Demarcação de estoma intestinal em adultos e idosos: evidências para a proposição de um protocolo. Tese (Livre-Docência) - Escola de Enfermagem de Ribeirão Preto, Universidade de São Paulo. Ribeirão Preto-SP.

Teles, A. A. S. et al. (2017). Physical, psychosocial changes and feelings generated by intestinal ostomy for the patient: integrative review. Revista de Enfermagem UFPE online, 11(2), 1062-1072. https://doi.org/10.5205/1981-8963-v11i2a13477p1062-1072-2017

Wiedenbein, L. et al. (2016). Assessment of rehabilitation needs in colorectal cancer treatment: Results from a mixed audit and qualitative study in Denmark. Acta Oncology. 55(6),705-711. https://www.ncbi.nlm.nih.gov/pubmed/26808254

World Council of Enterostomal Therapists (WCET). (2014). International Ostomy Guideline Recommendations. WCET Journal. 34(2). https://www.wcetn .org/as se ts/Publications/wcet_april-june_2014f\%20iog\%20recommandations.pdf

Wound, Ostomy, and Continence Nurses Society. (2010). Management of the patients with a fecal ostomy: Best Practice Guideline for Clinicians Journal Wound Ostomy Continence Nursing society. 37(6), 596-598. https://doi.org/10.1097/won.0b013e3181f97e37

Yu, Y. et al. (2017). The Impact of the predictive nursing education process on degree of comfort and Quality of Life for patients in the Oncology Department. Iran Journal Public Health. 46(9), 1231-1236. https://www.ncbi.nlm.nih.gov/pmc/articles/PMC5632325/ 